\title{
Efficacy of arthrocentesis and lavage for treatment of post-traumatic arthritis in temporomandibular joints
}

\author{
Joo-Young Park' ${ }^{1}$, Jong-Ho Lee Le,3 $^{2,4}$ \\ ${ }^{1}$ Department of Oral and Maxillofacial Surgery, Seoul National University Dental Hospital, \\ ${ }^{2}$ Department of Oral and Maxillofacial Surgery, School of Dentistry, Seoul National University, \\ ${ }^{3}$ Clinical Translational Research Center for Dental Science, Seoul National University Dental Hospital, \\ ${ }^{4}$ Oral Cancer Center, Seoul National University Dental Hospital, Seoul, Korea
}

\begin{abstract}
J Korean Assoc Oral Maxillofac Surg 2020;46:174-182)
Objectives: Joint injuries frequently lead to progressive joint degeneration that causes articular disc derangement, joint inflammation, and osteoarthritis. Such arthropathies that arise after trauma are defined as post-traumatic arthritis (PTA). Although PTA is well recognized in knee and elbow joints, PTA in the temporomandibular joint (TMJ) has not been clearly defined. Interestingly, patients experiencing head and neck trauma without direct jaw fracture have displayed TMJ disease symptoms; however, definitive diagnosis and treatment options are not available. This study will analyze clinical aspects of PTA in TMJ and their treatment outcomes after joint arthrocentesis and lavage.

Materials and Methods: Twenty patients with history of trauma to the head and neck especially without jaw fracture were retrospectively studied. Those patients developed TMJ disease symptoms and were diagnosed by computed tomography or magnetic resonance imaging. To decrease TMJ discomfort, arthrocentesis and lavage with or without conservative therapy were applied, and efficacy was evaluated by amount of mouth opening and pain scale. Statistical differences between pre- and post-treatment values were evaluated by Wilcoxon signed-rank test.

Results: Patient age varied widely between 20 and 80 years, and causes of trauma were diverse. Duration of disease onset was measured as 508 posttrauma days, and $85 \%$ of the patients sought clinic visit within 2 years after trauma. In addition, $85 \%$ of the patients showed TMJ disc derangement without reduction, and osteoarthritis was accompanied at the traumatized side or at both sides in $40 \%$ of the patients. After arthrocentesis or lavage, maximal mouth opening was significantly increased $(28-44 \mathrm{~mm}$ on average, $P<0.001)$ and pain scale was dramatically decreased $(7.8-3.5$ of 10 , $P<0.001)$; however, concomitant conservative therapy showed no difference in treatment outcome.

Conclusion: The results of this study clarify the disease identity of PTA in TMJ and suggest early diagnosis and treatment options to manage PTA in
\end{abstract} TMJ.

Key words: Temporomandibular joint disc, Synovial fluid, Joint diseases, Post-traumatic headache, Osteoarthritis

[paper submitted 2020. 4. 3 / revised 2020. 4. 3 / accepted 2020. 4. 4]

\section{Introduction}

The temporomandibular joint (TMJ) is the only joint in head and neck and hinges the mandible to the temporal bone of the skull. The TMJ is in near constant motion for swallow-

\footnotetext{
Joo-Young Park

Department of Oral and Maxillofacial Surgery, Seoul National University Dental Hospital, 101 Daehak-ro, Jongno-gu, Seoul 03080, Korea

TEL: +82-2-2072-4498 FAX: +82-2-766-4948

E-mail:bbyoung1@snu.ac.kr

ORCID: https://orcid.org/0000-0002-0333-6349
}

(2) This is an open-access article distributed under the terms of the Creative Commons Attribution Non-Commercial License (http://creativecommons.org/ licenses/by-nc/4.0/), which permits unrestricted non-commercial use, distribution, and reproduction in any medium, provided the original work is properly cited. Copyright (C) 2020 The Korean Association of Oral and Maxillofacial Surgeons. All rights reserved. ing, talking, yawning, and breathing and is one of the most frequently used joints in the body. The TMJ is a very delicate joint in structure and function ${ }^{1-3}$. Importantly, TMJ has an articular disc, a fibrocartilage tissue that runs between the fossa of the temporal bone above and the condyle of the mandible below. Because the TMJ disc provides a low friction surface and allows smooth articulation, displacement of the disc can result in joint noise, trismus, pain, or discomfort in the $\mathrm{TMJ}^{4-6}$. Combined dysfunction of the TMJ is defined as temporomandibular joint disorder (TMD) and comprises two major symptoms, TMJ pain and physical dysfunction including mouth opening limitation ${ }^{1-3}$.

Direct and indirect head trauma can affect TMJ, and fractures in the mandibular condyle especially have been considered as an etiology of chronic $\mathrm{TMD}^{7-12}$. Joint injuries 
frequently lead to progressive joint degeneration that causes articular disc dislocation, synovitis, and osteoarthritis ${ }^{13,14}$. As osteoarthritis is the final stage of arthropathy, post-traumatic arthritis (PTA) is defined as a condition triggered by acute joint trauma that can lead to osteoarthritis or chronic inflammatory arthropathies ${ }^{13,14}$. Pathologic mechanisms of PTA have been well described especially in the knee joint and symptoms include swelling, synovial effusion, pain, and sometimes intra-articular bleeding ${ }^{15-17}$. Usually, PTA recovers spontaneously, but persistence of symptoms after 6 months may be considered pathological and so-called chronic PTA $^{13,14,18}$.

A variety of molecular, mechanobiological, and cellular events involved in the pathogenesis and progression of PTA have been identified. Activation of inflammatory mechanisms including proinflammatory cytokine production during the acute phase of PTA plays a critical role in chronic disease onset $^{13,19}$. Human studies and experimental models have revealed that a series of inflammatory mediators is released in synovial fluid immediately after joint trauma ${ }^{17,20-23}$. These molecules have been proposed as markers of disease and as a potential target for specific and preventative interventions. However, chronic PTA cannot be prevented currently, although large numbers of agents have been tested in preclinical studies ${ }^{18,24-26}$.

Although PTA has been studied in many joints such as knee, elbow, ankle, and finger, PTA in TMJ has never been clearly defined. TMD arises after head and neck trauma even without direct mandibular fracture, and TMJ pain and dysfunction occur after head and neck trauma ${ }^{27-31}$. However, PTA in TMJ has not been familiar to clinicians, and the exact incidence and long-term consequences as well as a definitive treatment are not available.

In this study, patients experiencing indirect trauma history in the head and neck without jaw fracture and who developed TMD symptoms are retrospectively studied. Those patients complained of pain, mouth opening limitation, chewing difficulties, malocclusion, or joint noise combined with TMJ disc derangement without reduction or joint arthritis, as verified on computed tomography (CT) or magnetic resonance imaging (MRI). To decrease patient discomfort, arthrocentesis and lavage with or without conservative therapy were performed, and efficacy of the treatments was investigated for possible guidelines for treatment of PTA in TMJ.

\section{Materials and Methods}

\section{Patients and chart review}

A total of 20 patients diagnosed with TMD at the Department of Oral and Maxillofacial Surgery at Seoul National University Dental Hospital, Seoul, South Korea, during May 2018 to April 2020 was included in this study. This study was approved by the Seoul National University Dental Hospital Institutional Review Board (IRB approval No. ERI19015). Inclusion criteria were (1) exposure to well-defined recent or previous head and neck trauma; (2) clinical signs and symptoms corresponding to TMD, such as mouth opening limitation, deviation of mouth opening path, joint and muscle pain, headache, and joint noise; (3) and underwent TMJ CT or MRI. Exclusion criteria were (1) history of any TMJ surgery and (2) previous jaw fracture due to trauma including the maxilla or mandible.

For selected patients, etiology, date of trauma, patient age, date of first visit to the TMJ clinic, and TMD symptoms were retrospectively investigated by chart review. Disc derangement was diagnosed by MRI, and existence of osteoarthritis was diagnosed by TMJ radiographs and CT scan. Amount of maximal mouth opening (MMO, mm) was measured, and score of numerical rating scale (NRS; 0: no pain, 10: maximal pain) was recorded for pain at every patient visit.

\section{Arthrocentesis and lavage}

Arthrocentesis and lavage were performed in patients as the first choice of treatment by one oral and maxillofacial surgeon with or without conservative therapy. Conservative therapy included physical and occlusal stabilizing splint therapy provided at the department of oral medicine at the same hospital. The specific techniques of arthrocentesis and lavage were as previously published ${ }^{32}$. Briefly summarized, the ear and preauricular skin over the TMJ were prepared with topical antiseptic solution. Two injection points were marked over the articular fossa and eminence, 1 and $2 \mathrm{~cm}$ in front of the tragus along the canthal-tragus line. The auriculotemporal nerve was blocked with local anesthetics, and a 21-gauge needle with $10 \mathrm{~mL}$ syringe (BD Biosciences, San Jose, CA, USA) was introduced into the superior joint space. Approximately $2 \mathrm{~mL}$ of sterile normal saline solution was injected to distend the superior joint space. Occasionally, a second 21-gauge needle was inserted when the resistance of intra-joint space pressure was high. A total of 30-50 mL of 
normal saline was used for lavage. Patient mandibles were manipulated by the surgeon in a vertical direction for joint space enlargement. Then, $1 \mathrm{~mL}$ of steroid (Dexamethasone 5 mg; Daewon Pharmaceutical, Seoul, Korea) or 1-1.5 mL of sodium hyaluronate (Hanmi Pharmaceutical, Seoul, Korea) was injected into the joint space after lavage. Medication injection was followed by the authors's own protocol. Corticosteroid was used primarily for the first and second visits when synovial fluid was inflamed, while at the third visit and thereafter when the synovial fluid became clear, sodium hyaluronate was injected.

\section{Statistical analyses}

The age distribution was evaluated and passed the normality test by the one-sample Kolmogorov-Smirnov test. Differences in treatment outcomes before and after treatments were assessed using the Wilcoxon signed rank test. Changes in $\mathrm{MMO}$ and pain scales were compared between the two groups by Mann-Whitney test. All reported $P$-values were based on 2-sided tests, and statistical significance was marked as $* P<0.01, * * P<0.05, * * * P<0.001$, or not significant. Statistical analyses were performed with GraphPad Prism 8.2.1 (GraphPad Software, San Diego, CA, USA).

\section{Results}

\section{Clinical aspects of PTA in TMJ}

The ages of the 20 patients varied widely between 20 and 80 years, and mean age was 48.7 years.(Fig. 1. A; left panel) The age distribution passed the normality test $(\alpha>0.05)$ by the one-sample Kolmogorov-Smirnov test $(P>0.1)$, indicating no age prevalence in PTA development among the 20 patients included in this study.(Fig. 1. A; right panel) Interestingly, there was no skew in sex ratio (male=9, female=11) (Fig. 1 . $\mathrm{B})$, which is a conflicting result compared to general TMD development, where female patients show two times greater risk than male patients ${ }^{33}$. The causes of trauma were diverse, as traffic accident $(n=6)$, assault $(n=3)$, hit by materials $(n=3)$, and fall-down $(\mathrm{n}=8)$. All causes had similar frequencies.(Fig. 1. C)

While collecting patient history, the author surgeons noticed that patients with head and neck trauma without direct jaw fractures easily ignored their initial TMJ symptoms such as mild preauricular pain and mouth opening limitation and often did not seek medical attention. However, they had to seek doctors at later time points but the TMD progressed into chronic stage or irreversible osteoarthritis. Therefore, the authors wondered about the duration of disease onset for PTA in TMJ patients and, specifically, at which point the TMD symptoms increased and were reported to a doctor. To answer
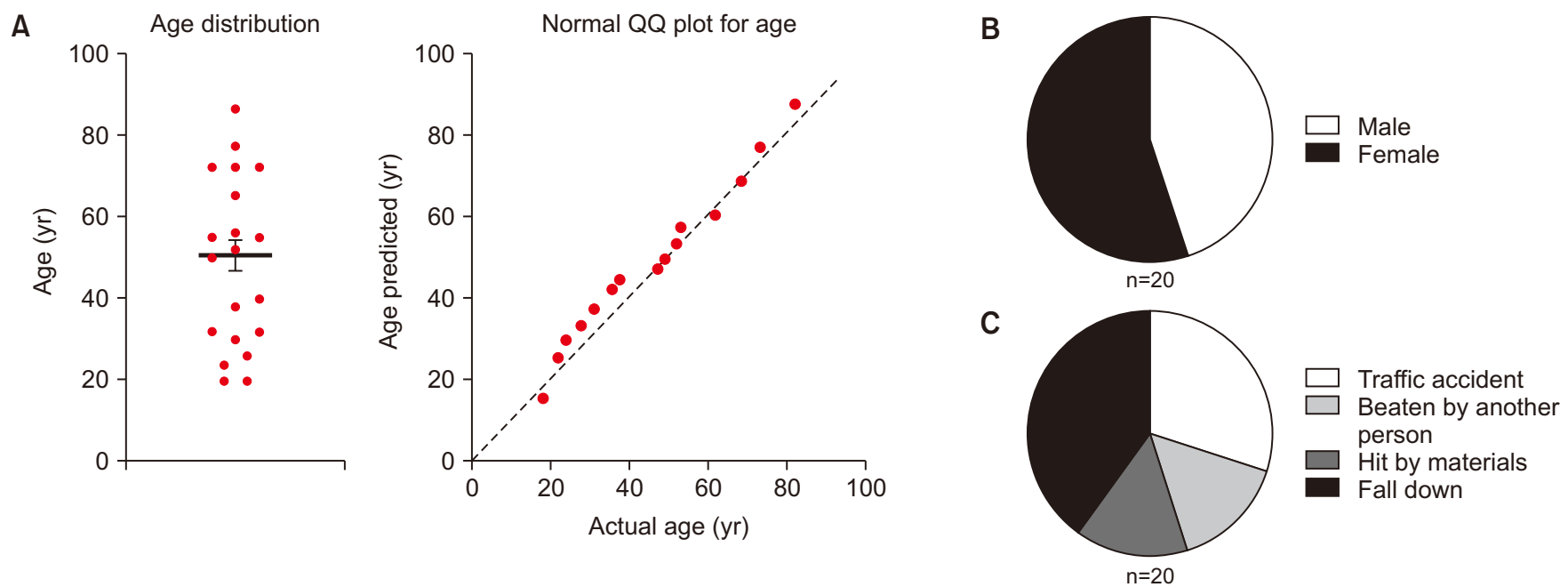

Fig. 1. Clinical aspects of post-traumatic arthritis in temporomandibular joint. A. Age distribution of the 20 patients. Mean age was 48.7 years (thick bar in the middle) with standard error of 4.6 years (left panel). The age distribution passed the normality test ( $\alpha>0.05)$ by the one-sample Kolmogorov-Smirnov test $(P>0.1)$, and the normal distribution is shown in a $Q Q$ plot on the right panel. B. Sex distribution of the patients (male=9, female=11). C. The causes of trauma of the 20 patients were diverse, as comprising traffic accident $(n=6)$, assault $(n=3)$, hit by materials $(n=3)$, and fall down $(n=8)$.

Joo-Young Park et al: Efficacy of arthrocentesis and lavage for treatment of post-traumatic arthritis in temporomandibular joints. J Korean Assoc Oral Maxillofac Surg 2020 
A

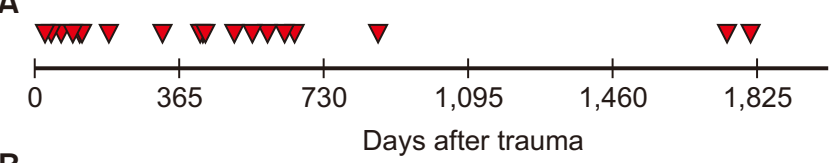

B

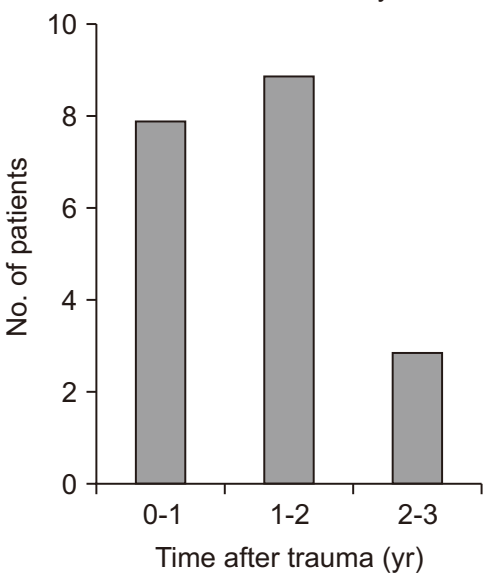

Fig. 2. Onset of post-traumatic arthritis (PTA) in temporomandibular joint (TMJ). A. When a PTA patient reports to a clinic with TMJ discomfort, the time since initial trauma should be measured. Days after trauma was calculated as the duration between initial trauma and the patient's first visit to the clinic and is was marked with a triangle in the bar graph. The average "days after trauma" was 508 days, with a standard error of 112 days. B. The bar graphs show the number of PTA patients reporting to the TMJ clinic during the 1 st $(n=8)$, 2nd $(n=9)$, or 3rd $(n=3)$ year after initial trauma. In total, $85.0 \%$ of the patients (17 of 20 ) sought help within 2 years after trauma.

Joo-Young Park et al: Efficacy of arthrocentesis and lavage for treatment of posttraumatic arthritis in temporomandibular joints. J Korean Assoc Oral Maxillofac Surg 2020

these questions, duration after trauma was measured. The average was 508 post-trauma days since the trauma until first visit to the clinic.(Fig. 2. A) In addition, $85.0 \%$ of the patients (17 of 20) sought clinic visit within 2 years after trauma.(Fig. 2. B)

\section{Diagnosis of TMJ arthritis in PTA patients}

TMJ MRI showed TMJ disc derangement without reduction in $85.0 \%$ of the patients (17 out of 20 ), 5 of whom showed derangement at both sides.(Fig. 3) Further, 40.0\% of the patients ( 8 of 20) suffered osteoarthritis at the traumatized side of the TMJ, 2 of whom had bilateral osteoarthritis. Only 2 patients were diagnosed with disc derangement with reduction. One patient was clinically diagnosed with fibrous ankylosis of both TMJs, with symptoms of mouth opening limitation and chewing difficulty without evidence of disc derangement on CT and MRI.(Fig. 3)



Fig. 3. Diagnosis of temporomandibular joint (TMJ) arthritis in post-traumatic arthritis patients by computed tomography or magnetic resonance imaging (MRI). TMJ MRI showed TMJ disc derangement without reduction (DD w/o reduction) in $85 \%$ of the patients (17 of 20), 5 of whom showed derangement at both sides. Eight patients suffered degenerative joint disease (DJD) such as osteoarthritis at the traumatized side of the TMJ, 2 of which had osteoarthritis at both sides. Only 2 patients were diagnosed with disc derangement with reduction (DD w/ reduction). Joo-Young Park et al: Efficacy of arthrocentesis and lavage for treatment of posttraumatic arthritis in temporomandibular joints. J Korean Assoc Oral Maxillofac Surg 2020

\section{Treatment efficacy of joint arthrocentesis and lavage in PTA patients}

All patients received joint arthrocentesis or lavage with or without conservative therapy.(Fig. 4. A) After arthrocentesis or lavage, improvement of MMO was statistically significant (28-44 mm on average, $P<0.001$ ) (Fig. 4. B), and there was a dramatic reduction of pain scale (7.8-3.5 of $10, P<0.001)$. (Fig. 4. C)

To develop a standard protocol of joint arthrocentesis for PTA patients in clinics, the authors wanted to know the numbers of performances and days of lavage which are required to resolve limited mouth opening and pain. Mechanistically, numbers of treatment days and visits until mouth opening reached $40 \mathrm{~mm}$ were calculated. The results were an average of 153 days (standard error [SE], \pm 35 days) and 3.2 rounds (SE, \pm 0.4 rounds) of arthrocentesis to reach $40 \mathrm{~mm}$ of MMO.

(Fig. 5. A, 5. B)

The authors' next question was whether concomitant conservative therapy can increase the efficacy of lavage for PTA patients. Conservative therapy consists of physical therapy, e.g., moist hot pack, ultrasound, massage, and occlusal stabilizing splint therapy, and 8 of 20 patients were treated concomitantly with joint lavage.(Fig. 4. A) From a pathophysiological viewpoint, PTA develops initially as acute 
A

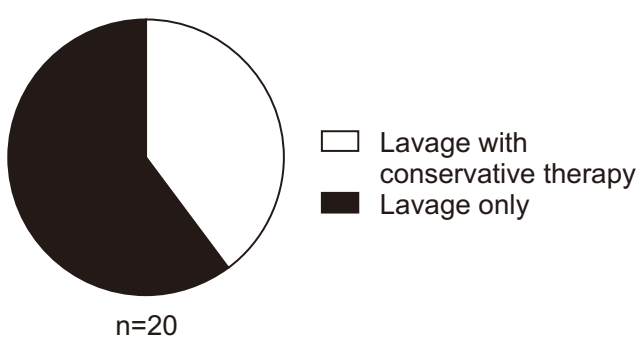

B



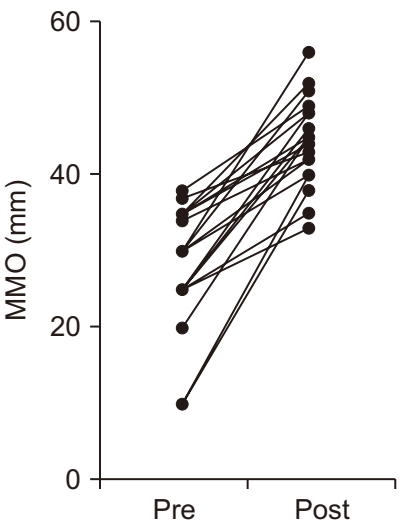

C



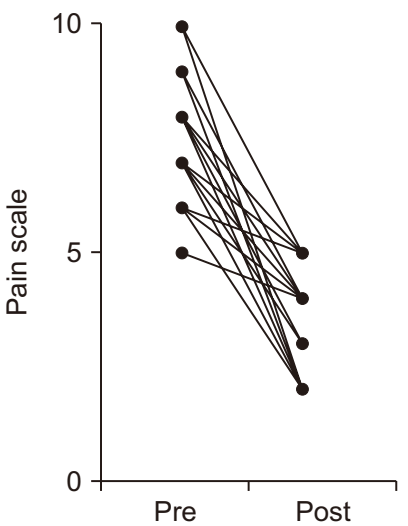

Fig. 4. Treatment efficacy of temporomandibular joint arthrocentesis and lavage in post-traumatic arthritis patients. A. All patients received joint arthrocentesis or lavage with $(n=8)$ or without $(n=12)$ conservative therapy. B. After arthrocentesis or lavage, improvement of maximal mouth opening (MMO) was statistically significant (28-44 mm in average, ${ }^{* \star}{ }^{*} P<0.001$, left panel). The dot plot shows amount of $\mathrm{MMO}$ at pre- or post-treatment visit. Thick black bar shows the average and standard error of 20 patients. Pre- and post-lavage MMOs in the same patient were connected as paired data (right panel). C. Pain scale was dramatically reduced from 7.8 to 3.5 of $10\left({ }^{* * *} P<0.001\right)$. The dot plot shows the pain scale at pre- or post-treatment visit. Thick black bar shows average and standard error of 20 patients. The pain scales at pre- and post-lavage in the same patient were connected as paired data (right panel). Differences in treatment outcomes before and after treatments were assessed using the Wilcoxon signed rank test. All reported $P$-values were based on two-sided tests, and statistical significance was shown as ${ }^{\star \star \star} P<0.001$.

Joo-Young Park et al: Efficacy of arthrocentesis and lavage for treatment of post-traumatic arthritis in temporomandibular joints. J Korean Assoc Oral Maxillofac Surg 2020

joint inflammation due to trauma, and such pathological progress can be reversed by resolution of inflammation by lavage without conservative therapy. To verify this, changes in MMO and pain scales were compared between a lavage only group and a lavage with conservative therapy group. Concomitant conservative therapy showed no statistically significant difference in treatment outcome in PTA patients. (Fig. 5. C)

\section{Discussion}

TMJ is the only joint where 2 separate joints being connected in a bone (mandible) and should move properly with coordination. However, imbalance of any part of the joint can cause deterioration and result in disease states of one or both sides of joint. Head and neck trauma can deteriorate TMJ balance, and trauma with jaw fracture is an etiology of chronic
$\mathrm{TMD}^{8,12,29,34,35}$. The exact incidence of TMD after trauma and long-term consequences of TMD have not been well supported with evidence. A meta-analytic observational study based on literature review found that TMJ imbalance with joint clicking was reported in $15.9 \%$ of patients undergoing closed treatment, whereas patients treated by open reduction showed $10.3 \%$ incidence $^{34}$. A retrospective study in 2014 reported that condylar fracture associated with concomitant angle/body fracture showed a high incidence of TMD issues ${ }^{36}$. Because of lack of systematic evidence-based studies with long-term follow-up and complex etiology, it is difficult to ascertain the details of the cause-effect relationship of mandibular trauma and TMD.

Despite of the ambiguity and complexity of post-traumatic TMD, patients who experienced recent or old head and neck trauma even without direct jaw fracture often report to clinics with complaint of discomfort such as preauricular pain and 
A

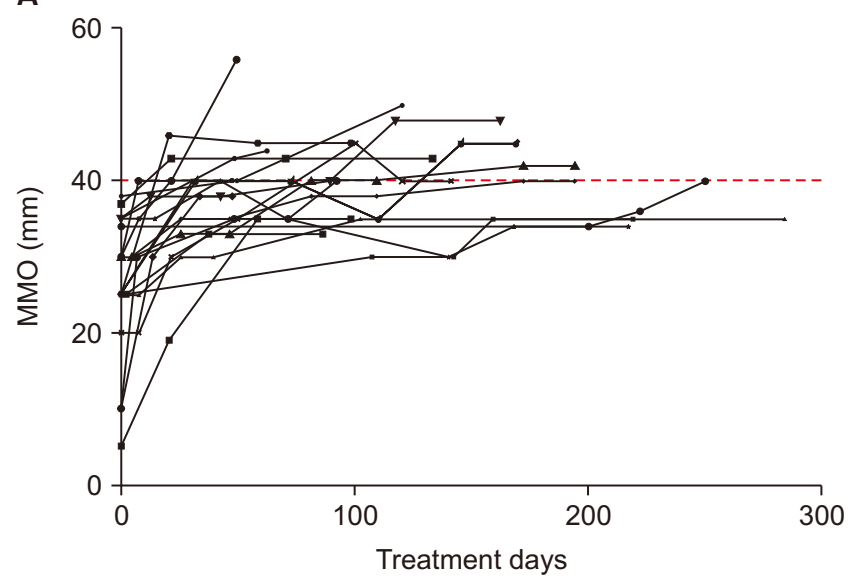

B

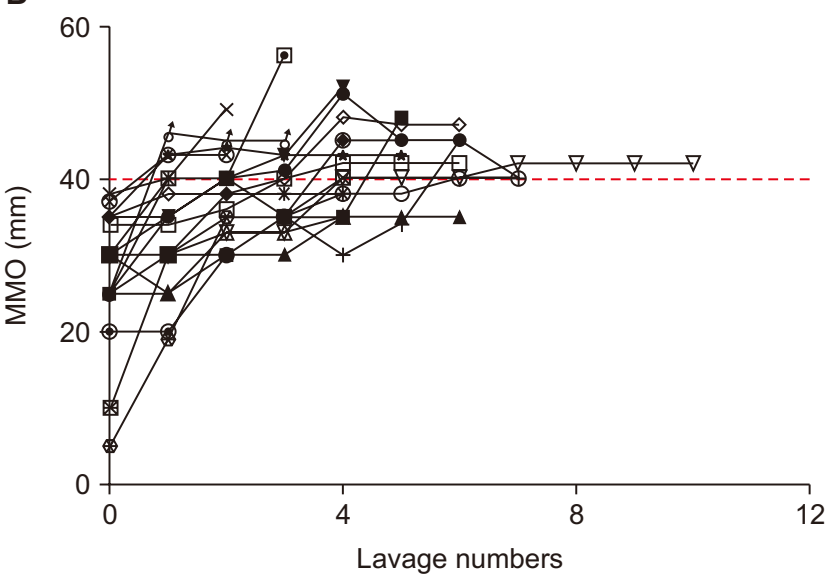

\section{C}

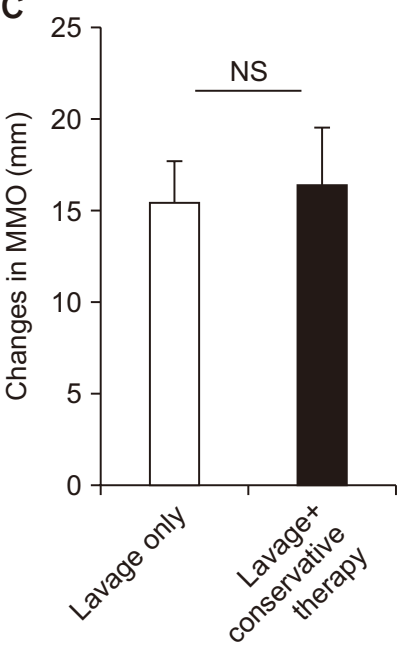

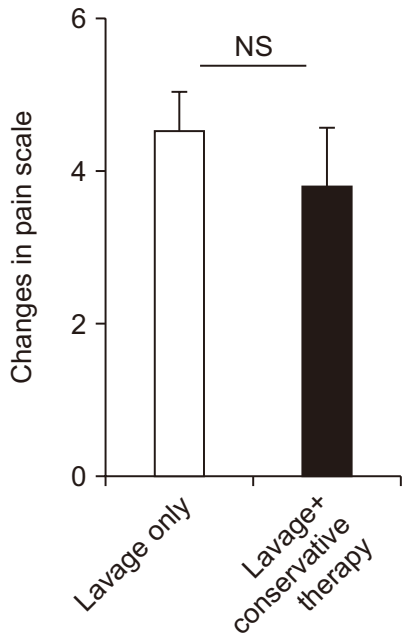

Fig. 5. Recommended treatment protocol for post-traumatic arthritis in temporomandibular joint. A. Maximal mouth opening (MMO, mm) over treatment days, where day "O" is the first visit at the clinic. The red dotted line represents $40 \mathrm{~mm}$ of mouth opening. B. MMO over treatment numbers. The $\mathrm{x}$-axis is the number of patients' visits. The red dotted line represents $40 \mathrm{~mm}$ of mouth opening. Each dot represents one patient's visit and each identical figure represents one patient. C. Comparison of pre- and post-treatment MMO (left panel) and pain scale (right panel) between the two groups: lavage only group versus lavage with concomitant conservative therapy group. Bar graphs show average changes in MMO with standard errors. Changes in MMO and pain scales were compared between the two groups by Mann-Whitney test. (NS: not significant)

Joo-Young Park et al: Efficacy of arthrocentesis and lavage for treatment of post-traumatic arthritis in temporomandibular joints. J Korean Assoc Oral Maxillofac Surg 2020

mouth opening limitation. Interestingly, whiplash neck trauma induced by rapid back-and-forth movement of the neck has association with $\mathrm{TMD}^{28,31,35,37}$. This implies that TMD can result from indirect head and neck trauma.

In the pathophysiology of post-traumatic arthritis, PTA is well known in knee, elbow, and ankle joints ${ }^{14,15,24,38}$. PTA accounts for about $12 \%$ of all osteoarthritis cases in orthopedic clinics, and a history of physical trauma may also be found in patients with chronic inflammatory arthritis in knees ${ }^{38}$. The acute symptoms following joint trauma include swelling, synovial effusion, severe pain, and occasional intra-joint bleeding. Usually, PTA recovers spontaneously in 2-3 months ${ }^{13}$. Persistence of symptoms after this period deserve attention; a duration of 6 months or longer may be considered pathologi- cal and so-called chronic PTA. Chronic PTA represents an inflammatory condition that persists over time after a joint trauma. The most frequent chronic PTA is post-traumatic osteoarthritis (PTOA) $)^{13,16,38,39}$.

Joint tissue injury can lead to increase of both matrix molecules and complement components released into the synovial fluid. Some matrix molecules may act as Toll-like receptor (TLR) agonists, while others can activate the complement cascade. Both can activate secretion of pro-inflammatory cytokines and chemokine production from synovial membrane cells, which can promote cellular infiltration, the hallmark of synovitis ${ }^{19,39,40}$. This molecular and cellular inflammation can potentiate cartilage erosion via production of enzymatic mediators of matrix degradation. There is a substantial body 
of evidence from multiple sources demonstrating a relationship between synovitis and symptoms such as pain, swelling, and joint dysfunction in knee osteoarthritis patients. Evidence from a smaller number of arthroscopic and imaging studies suggests that the presence of synovitis may be associated with faster rate of cartilage erosion in osteoarthritis. Although not yet demonstrated in patients, additional studies of animal models suggest that synovial macrophages, increased in synovitis, may be involved in osteophyte formation in osteoarthritis $^{20,41-43}$.

To eliminate inflamed synovial fluid, arthrocentesis and lavage were chosen as the primary treatment option in this study. After arthrocentesis, among the various medications available, sodium hyaluronate and corticosteroids were used in this study. The anti-inflammatory effects of intra-articular corticosteroids on synovial tissues have been well documented, and these drugs are useful for reducing pain and dysfunction in patients with inflammatory diseases of the joints. However, side effects of intra-articular injection of corticosteroids such as destruction of cartilage and progression of joint disease also have been reported. Therefore, dexamethasone was injected only at the first and second lavage, especially for pain alleviation and anti-inflammation ${ }^{44,45}$. To achieve a longterm effect of joint lubrication, sodium hyaluronate was used as intra-joint injection medication starting at the third lavage. Sodium hyaluronate is the sodium salt of hyaluronic acid, which is a polysaccharide of the glycosaminoglycans family, found in many extracellular tissues, including synovial fluid and cartilage. Intra-articular injection of sodium hyaluronate was reported to offer consistent benefit for at least 6 months, primarily in patients with disc displacement with reduction, and this was attributed to the mechanical effect of this medication $^{45-47}$.

The authors of the present study aimed to elucidate the clinical aspects of PTA developed in TMJ. Although the study was investigated in a retrospective manner with a limited number of chart reviews, several interesting findings about PTA in TMJ were noted. First, PTA in TMJ can develop regardless of age and sex of the patients and is caused by various types of head and neck trauma, different from the general characteristics of TMD, which shows female predominance. Secondly, most patients report to a TMJ clinic within 2 years after trauma with a complaint of PTA. This is important information for clinicians because head and neck trauma patients might require recall-checks until 2 years, even in patients with asymptomatic TMJ. Thirdly, we chose joint lavage as the first-line treatment option to resolve soluble pro-in- flammatory cytokines and eliminate inflamed synovial fluid. Efficacy of lavage in PTA was high in improvement of mouth opening and reduction of pain, in an average of 5 months, with more than 3 lavage treatments needed to recover mouth opening by $40 \mathrm{~mm}$. Lastly, concomitant conservative therapy may be adjunctive to joint lavage of arthrocentesis, although it is not essential to treat PTA in TMJ.

\section{Conclusion}

The results of this study clarify the disease identity of PTA in TMJ and provide data to suggest possible treatment guidelines and options to manage PTA in TMJ in clinics. Despite this essential information from the study, a case controlled prospective study with larger number of clinical cases of PTA in TMJ is necessary to answer many important questions, such as the incidence of PTA in TMJ among head and neck trauma patients. Inversely, clinicians do not know the frequency of TMD due to head and neck trauma or the best treatment option for those PTA patients. Those answers will be cornerstones for future TMJ studies.

\section{ORCID}

Joo-Young Park, https://orcid.org/0000-0002-0333-6349

Jong-Ho Lee, https://orcid.org/0000-0002-8843-545X

\section{Authors' Contributions}

J.Y.P. designed the study, collected and analyzed data and wrote the manuscript. J.H.L. designed the study, participated in data collection, and reviewed the manuscript. All authors read and approved the final manuscript.

\section{Ethics Approval and Consent to Participate}

This study was performed after receiving ethics approval from the Seoul National University Dental Hospital Institutional Review Board (IRB approval No. ERI19015). Data were obtained by retrospective chart review and do not include personal identification; thus, the need for obtaining informed consent from subjects was waived by the IRB.

\section{Conflict of Interest}

No potential conflict of interest relevant to this article was reported. 


\section{References}

1. Ahmad M, Schiffman EL. Temporomandibular joint disorders and orofacial pain. Dent Clin North Am 2016;60:105-24.

2. Roberts WE, Stocum DL. Part II: temporomandibular joint (TMJ)regeneration, degeneration, and adaptation. Curr Osteoporos Rep 2018;16:369-79.

3. Stocum DL, Roberts WE. Part I: development and physiology of the temporomandibular joint. Curr Osteoporos Rep 2018;16:360-8.

4. Barkin S, Weinberg S. Internal derangements of the temporomandibular joint: the role of arthroscopic surgery and arthrocentesis. J Can Dent Assoc 2000;66:199-203.

5. Chisnoiu AM, Picos AM, Popa S, Chisnoiu PD, Lascu L, Picos A, et al. Factors involved in the etiology of temporomandibular disorders - a literature review. Clujul Med 2015;88:473-8.

6. Dolwick MF. Temporomandibular joint surgery for internal derangement. Dent Clin North Am 2007;51:195-208, vii-viii.

7. Krishnan DG. Soft tissue trauma in the temporomandibular joint region associated with condylar fractures. Atlas Oral Maxillofac Surg Clin North Am 2017;25:63-7.

8. Renapurkar SK, Strauss RA. Temporomandibular joint trauma. Atlas Oral Maxillofac Surg Clin North Am 2019;27:99-106.

9. Salé H, Isberg A. Delayed temporomandibular joint pain and dysfunction induced by whiplash trauma: a controlled prospective study. J Am Dent Assoc 2007;138:1084-91.

10. Takahashi T, Ohtani M, Sano T, Ohnuki T, Kondoh T, Fukuda M. Magnetic resonance evidence of joint effusion of the temporomandibular joint after fractures of the mandibular condyle: a preliminary report. Cranio 2004;22:124-31.

11. Tripathi R, Sharma N, Dwivedi AN, Kumar S. Severity of soft tissue injury within the temporomandibular joint following condylar fracture as seen on magnetic resonance imaging and its impact on outcome of functional management. J Oral Maxillofac Surg 2015;73:2379.e1-7.

12. Yun PY, Kim YK. The role of facial trauma as a possible etiologic factor in temporomandibular joint disorder. J Oral Maxillofac Surg 2005;63:1576-83.

13. Lieberthal J, Sambamurthy N, Scanzello CR. Inflammation in joint injury and post-traumatic osteoarthritis. Osteoarthritis Cartilage 2015;23:1825-34.

14. Punzi L, Galozzi P, Luisetto R, Favero M, Ramonda R, Oliviero F, et al. Post-traumatic arthritis: overview on pathogenic mechanisms and role of inflammation. RMD Open 2016;2:e000279.

15. Englund M, Guermazi A, Lohmander LS. The meniscus in knee osteoarthritis. Rheum Dis Clin North Am 2009;35:579-90.

16. Gelber AC, Hochberg MC, Mead LA, Wang NY, Wigley FM, Klag MJ. Joint injury in young adults and risk for subsequent knee and hip osteoarthritis. Ann Intern Med 2000;133:321-8.

17. Teunis T, Beekhuizen M, Van Osch GV, Schuurman AH, Creemers LB, van Minnen LP. Soluble mediators in posttraumatic wrist and primary knee osteoarthritis. Arch Bone Jt Surg 2014;2:146-50.

18. Barton KI, Shekarforoush M, Heard BJ, Sevick JL, Vakil P, Atarod $\mathrm{M}$, et al. Use of pre-clinical surgically induced models to understand biomechanical and biological consequences of PTOA development. J Orthop Res 2017;35:454-65.

19. Olson SA, Horne P, Furman B, Huebner J, Al-Rashid M, Kraus VB, et al. The role of cytokines in posttraumatic arthritis. J Am Acad Orthop Surg 2014;22:29-37.

20. Shen PC, Wu CL, Jou IM, Lee CH, Juan HY, Lee PJ, et al. Thelper cells promote disease progression of osteoarthritis by inducing macrophage inflammatory protein- $1 \gamma$. Osteoarthritis Cartilage 2011;19:728-36.

21. Takebe K, Rai MF, Schmidt EJ, Sandell LJ. The chemokine receptor CCR5 plays a role in post-traumatic cartilage loss in mice, but does not affect synovium and bone. Osteoarthritis Cartilage 2015;23:454-61.
22. Teunis T, Beekhuizen M, Kon M, Creemers LB, Schuurman AH, van Minnen LP. Inflammatory mediators in posttraumatic radiocarpal osteoarthritis. J Hand Surg Am 2013;38:1735-40.

23. van Meegeren ME, Roosendaal G, Jansen NW, Wenting MJ, van Wesel AC, van Roon JA, et al. IL-4 alone and in combination with IL-10 protects against blood-induced cartilage damage. Osteoarthritis Cartilage 2012;20:764-72.

24. Anderson DD, Chubinskaya S, Guilak F, Martin JA, Oegema TR, Olson SA, et al. Post-traumatic osteoarthritis: improved understanding and opportunities for early intervention. J Orthop Res 2011;29:802-9.

25. Caron JP, Fernandes JC, Martel-Pelletier J, Tardif G, Mineau F, Geng C, et al. Chondroprotective effect of intraarticular injections of interleukin-1 receptor antagonist in experimental osteoarthritis. Suppression of collagenase-1 expression. Arthritis Rheum 1996;39:1535-44.

26. Goodrich LR, Phillips JN, McIlwraith CW, Foti SB, Grieger JC, Gray SJ, et al. Optimization of scAAVIL-1ra in vitro and in vivo to deliver high levels of therapeutic protein for treatment of osteoarthritis. Mol Ther Nucleic Acids 2013;2:e70.

27. Fischer DJ, Mueller BA, Critchlow CW, LeResche L. The association of temporomandibular disorder pain with history of head and neck injury in adolescents. J Orofac Pain 2006;20:191-8.

28. Häggman-Henrikson B, Rezvani M, List T. Prevalence of whiplash trauma in TMD patients: a systematic review. J Oral Rehabil 2014;41:59-68.

29. Kim YK. Traumatic TMJ injury. J Korean Assoc Maxillofac Plast Reconstr Surg 1997;19:191-9.

30. Klobas L, Tegelberg A, Axelsson S. Symptoms and signs of temporomandibular disorders in individuals with chronic whiplashassociated disorders. Swed Dent J 2004;28:29-36.

31. Packard RC. The relationship of neck injury and post-traumatic headache. Curr Pain Headache Rep 2002;6:301-7.

32. Dimitroulis G, Dolwick MF, Martinez A. Temporomandibular joint arthrocentesis and lavage for the treatment of closed lock: a followup study. Br J Oral Maxillofac Surg 1995;33:23-6; discussion 26-7.

33. Bueno CH, Pereira DD, Pattussi MP, Grossi PK, Grossi ML. Gender differences in temporomandibular disorders in adult populational studies: a systematic review and meta-analysis. J Oral Rehabil 2018;45:720-9.

34. García-Guerrero I, Ramírez JM, Gómez de Diego R, MartínezGonzález JM, Poblador MS, Lancho JL. Complications in the treatment of mandibular condylar fractures: Surgical versus conservative treatment. Ann Anat 2018;216:60-8.

35. Pullinger AG, Seligman DA. Trauma history in diagnostic groups of temporomandibular disorders. Oral Surg Oral Med Oral Pathol 1991;71:529-34.

36. Tabrizi R, Bahramnejad E, Mohaghegh M, Alipour S. Is the frequency of temporomandibular dysfunction different in various mandibular fractures? J Oral Maxillofac Surg 2014;72:755-61.

37. Howard RP, Hatsell CP, Guzman HM. Temporomandibular joint injury potential imposed by the low-velocity extension-flexion maneuver. J Oral Maxillofac Surg 1995;53:256-62; discussion 263.

38. Brown TD, Johnston RC, Saltzman CL, Marsh JL, Buckwalter JA. Posttraumatic osteoarthritis: a first estimate of incidence, prevalence, and burden of disease. J Orthop Trauma 2006;20:739-44.

39. Kramer WC, Hendricks KJ, Wang J. Pathogenetic mechanisms of posttraumatic osteoarthritis: opportunities for early intervention. Int J Clin Exp Med 2011;4:285-98.

40. Honorati MC, Bovara M, Cattini L, Piacentini A, Facchini A. Contribution of interleukin 17 to human cartilage degradation and synovial inflammation in osteoarthritis. Osteoarthritis Cartilage 2002;10:799-807.

41. Bondeson J, Blom AB, Wainwright $\mathrm{S}$, Hughes $\mathrm{C}$, Caterson B, van den Berg WB. The role of synovial macrophages and macrophageproduced mediators in driving inflammatory and destructive responses in osteoarthritis. Arthritis Rheum 2010;62:647-57. 
42. Culemann S, Grüneboom A, Nicolás-Ávila JÁ, Weidner D, Lämmle KF, Rothe T, et al. Locally renewing resident synovial macrophages provide a protective barrier for the joint. Nature 2019;572:670-5.

43. Tu J, Hong W, Guo Y, Zhang P, Fang Y, Wang X, et al. Ontogeny of synovial macrophages and the roles of synovial macrophages from different origins in arthritis. Front Immunol 2019;10:1146.

44. Tozoglu S, Al-Belasy FA, Dolwick MF. A review of techniques of lysis and lavage of the TMJ. Br J Oral Maxillofac Surg 2011; 49:302-9.

45. Kopp S, Wenneberg B, Haraldson T, Carlsson GE. The short-term effect of intra-articular injections of sodium hyaluronate and corticosteroid on temporomandibular joint pain and dysfunction. J Oral Maxillofac Surg 1985;43:429-35.

46. Sequeira J, Rao BHS, Kedia PR. Efficacy of sodium hyaluronate for temporomandibular joint disorder by single-puncture arthrocentesis. J Maxillofac Oral Surg 2019;18:88-92.

47. Bertolami CN, Gay T, Clark GT, Rendell J, Shetty V, Liu C, et al. Use of sodium hyaluronate in treating temporomandibular joint disorders: a randomized, double-blind, placebo-controlled clinical trial. J Oral Maxillofac Surg 1993;51:232-42.

How to cite this article: Park JY, Lee JH. Efficacy of arthrocentesis and lavage for treatment of post-traumatic arthritis in temporomandibular joints. J Korean Assoc Oral Maxillofac Surg 2020;46:174-182. https://doi.org/10.5125/jkaoms.2020.46.3.174 